\title{
Low levels of $\mathrm{Na}$, K-ATPase and carbonic anhydrase II during choroid plexus development suggest limited involvement in early CSF secretion
}

\author{
Pia Johansson ${ }^{\mathrm{a},{ }^{*}, \text { Katarzyna Dziegielewska }}{ }^{\mathrm{a}}$, and Norman Saunders $\mathrm{a}, \mathrm{b}$ \\ a Department of Pharmacology, University of Melbourne, Parkville, Victoria 3010, Australia \\ b Centre for Neuroscience, University of Melbourne, Parkville, Victoria 3010, Australia
}

\section{Abstract}

In the adult, cerebrospinal fluid (CSF) is produced by the actions of numerous transporters and enzymes creating ion gradients that drive the entry of water into the ventricles via the aquaporin-1 water channels (AQP1). It is not known when in development CSF secretion starts but, in the rat, it has been postulated to occur around the time of birth. However, recent evidence suggests that the secretion may start much earlier, as soon as the lateral choroid plexuses first appear (around E14).

Purpose-To investigate the developmental profiles of two major enzymes responsible for CSF secretion in the adult, Na, K-ATPase (NKA) and carbonic anhydrase II (CAII).

Methods-The developmental profiles of both enzymes were investigated using immunohistochemistry and Western Blot analysis in tissue from embryonic day $(E) 15,18$, postnatal day $(P) 0,9$ and adult rats.

Result-Western Blot analysis showed low levels of NKA at E15 followed by a progressive increase with age. Immunohistochemistry confirmed the presence of NKA on the apical membrane of the lateral ventricular choroid plexus epithelium from E15 onwards. Western Blot analysis of CAII was complicated by its presence in blood, but the amount of protein increased with age.

Immunohistochemically, CAII appeared in the lateral ventricular choroid plexus between P0 and P9.

Conclusions-The low levels of NKA and CAII during early choroid plexus development indicate that other mechanisms, such as the previously described specific protein transfer across epithelial cells, may be involved in early CSF secretion and movement of water into the cerebral ventricles.

\section{Keywords}

Cerebrospinal fluid; Embryo; Brain development; Ventricular expansion; Epithelial cells

\begin{abstract}
In the adult, the majority of cerebrospinal fluid (CSF) is secreted into the cerebral ventricles by the epithelial cells of the four choroid plexuses [6]. A multitude of transporters and enzymes are responsible for the net movement of $\mathrm{Na}^{+}, \mathrm{HCO}^{3-}, \mathrm{Cl}^{-}$and water from blood into CSF and a net movement of $\mathrm{K}^{+}$in the opposite direction [4]. The ion gradients thus formed drive the entry of water into the CSF, mainly through the aquaporin-1 water channels (AQP1 [4,22]). Two main enzymes involved in this process are Na, K-ATPase (NKA) and carbonic anhydrase II (CAII) and the inhibition of either result in significant reductions in CSF secretion rate [4].
\end{abstract}


The NKA pump is widespread in secretory epithelia, but in contrast to its more common localisation on the basolateral surface, it is located on the apical membrane of adult choroid plexus epithelial cells $[20,25]$. The developmental studies of NKA have mainly been focused on its enzymatic activity and demonstrated its presence in the perinatal period in rats and rabbits $[21,23]$. The period of brain development when the choroid plexus first appears has not been investigated previously.

Several subtypes of carbonic anhydrases have been identified in the choroid plexus [5] but the cytosolic CAII is the most influential on CSF secretion. In development, CAII protein has been found in rat choroid plexus from E15 [19] and in humans from 7 to 9 weeks gestation [5,17]. However, the enzyme may not be functionally active as no effect on secretion was detected in response to the inhibitor acetazolamide in newborn rats [2].

The mechanisms of CSF secretion have been well described in the adult, but much less is known early in choroid plexus development. Studies measuring ion gradients and tracer clearance have estimated the start of secretion in the rat to around the time of birth [1-3,16]. More recent evidence suggests that fluid secretion starts much earlier in development, as soon as the choroid plexuses appear. In the rat the lateral ventricular choroid plexus first appears around E14 [8, 13] and the aquaporin- 1 water channel is present in the choroid plexus epithelial cells, in an adult-like pattern, from E15 [13]. The presence of AQP1 at E15 strongly suggests CSF formation at this age. This is further supported by the observed expansion of the lateral ventricles immediately after the choroid plexuses first appear [12]. This occurs at a time in development when the pores in roof of the fourth ventricle have not yet formed [15] and the water added to the ventricular space at this time cannot escape and thus contributes to the pressure required for ventricular expansion and proper brain development [7].

Thus it is likely that fluid secretion by the choroid plexus occurs during early stages of brain development, but it is not known if the mechanisms responsible for water entry from blood into the ventricles are the same as in the adult or if there are other developmental-specific systems involved. We have previously suggested that the high CSF protein concentration and specific protein transfer present early in choroid plexus development may be involved in driving CSF formation and ventricular expansion $[12,14]$. The aim of the present study was to investigate the ontogeny of NKA and CAII in the lateral ventricular choroid plexus in the rat in order to determine if these enzymes also play a role in CSF secretion during early brain development.

All animal experiments were conducted according to NHMRC guidelines and approved by the University of Melbourne Animal Experimentation Ethics Committee. Time-mated females at different gestational ages and postnatal Sprague-Dawley rats were used. All animals were terminally anaesthetised with an intraperitoneal injection of urethane (Sigma, 25\% (w/v), $1 \mathrm{ml} /$ $100 \mathrm{~g}$ bodyweight). The day of discovery of the overnight plug was counted as embryonic day $(E) 0$ and the day of birth as postnatal day $(P) 0$. The developmental stages of the embryos were confirmed by their weight and crown-rump length.

Lateral ventricular choroid plexuses from E15, E18, P0, P9 and adult rats were carefully dissected out in cold phosphate buffered saline (PBS), immediately immersed into a PBS with protein inhibitor (Complete Mini, Roche) and kept on ice. Both lateral ventricular choroid plexuses from approximately 6 embryos were pooled at E15, 3 at E18, 2 neonates at P0, 2 animals at $\mathrm{P} 9$ and from 1 adult. Three pooled samples from each age were used $(n=3)$.

Protein extraction was performed using the ProteoExtract Subcellular Proteome Extraction Kit (Calbiochem) according to the manufacturer's instructions but downscaled 10 times. Fraction 1 (cytosolic fraction) was used for CAII and fraction 2 (membrane fraction) was used for the assessment of NKA content. Protein content was measured using the standard Bradford Assay. 
The same amount of total protein (about $3.5 \mu \mathrm{g}$ ) was loaded onto each well ( $20 \mu \mathrm{l}$ wells, $12 \%$ $\mathrm{HCl}$-Tris Pre-Cast gels, BioRad) and separated using electrophoresis (Mini-Protean II, BioRad). The proteins were transferred onto a PVDF membrane (BioRad) using wet transfer. The PVDF membrane was blocked overnight at $4{ }^{\circ} \mathrm{C}$ in a solution containing $50 \%$ soymilk in tris-buffered saline (TBS) and $0.2 \%$ Tween.

The membrane was incubated with primary antibodies against Na, K-ATPase $\alpha_{1}$-subunit (NKA $\alpha_{1}, 1: 2000$ dilution, NB300-146, Novus Biologicals) or carbonic anhydrase II (1:400 dilution, sc-48351, Santa Cruz) and $\beta$-actin (1:3000 dilution, Sapphire BioSciences) in blocking solution (25\% soymilk in TBS and $0.1 \%$ Tween) for $1.5 \mathrm{~h}$ at room temperature followed by $3 \times 10 \mathrm{~min}$ washes in TBS. The membrane was incubated for $1.5 \mathrm{~h}$ with a rabbit anti-mouse secondary antibody (1:400 dilution, DAKO), washed (as above) and then incubated with a peroxidise-anti-peroxidase (PAP) conjugated anti-mouse tertiary antibody (1:400 dilution, DAKO) for $1.5 \mathrm{~h}$. Following a subsequent wash the antibody complexes were visualised using 3,3'-diaminobenzidine tetrahydrochloride (DAB, Sigma), the reaction was stopped by immersion of the membrane in distilled water after which the membrane was dried and photographed.

Brains were dissected out from E15, E18, P0 and P9 terminally anaesthetised Sprague-Dawley rats and immediately immersed in Bouin's fixative ( $n=3$ per age). Adult animals were first perfused-fixed with $4 \%$ paraformaldehyde in phosphate buffer after which the brains were dissected out and immersed in Bouin's fixative $(n=3)$. The specimens were dehydrated with graded alcohols, cleared in xylene and embedded in paraffin wax (Merck, melting point $52^{\circ}$ C). Serial sections, $5 \mu \mathrm{m}$ thick, were cut in coronal orientation and placed on silanized slides. Representative sections of each series were stained with haematoxylin and eosin for routine histology.

Paraffin embedded sections were dewaxed by heating $\left(60^{\circ} \mathrm{C}\right.$ for $\left.20 \mathrm{~min}\right)$ followed by histolene treatment and then rehydrated by a series of graded ethanol. Sections were incubated in Peroxidase Blocker (DAKO) to remove any endogenous peroxidase activity, followed by incubation in Protein Blocker (DAKO). The primary antibody (anti-Na, K-ATPase $\alpha_{1}$-subunit, 1:500 dilution, NB300-146, Novus Biologicals or anti-carbonic anhydrase II 1:400 dilution, sc-48351, Santa Cruz) was left on the sections overnight at $4{ }^{\circ} \mathrm{C}$. The overnight incubation was followed by $3 \times 5$ min washes in PBS containing $0.1 \%$ Tween-20 (Sigma). Subsequently, two incubations in rabbit anti-mouse immunoglobulins (1:200 dilution, DAKO) and PAP conjugated anti-mouse tertiary antibody (1:200 dilution, DAKO) were performed at room temperature for $2 \mathrm{~h}$ each. Each incubation was followed by three washes in PBS/Tween-20 buffer. The peroxidase reaction was developed in DAKO 3,3'-diaminobenzidine tetrahydrochloride liquid substrate solution (DAKO) for $5 \mathrm{~min}$ and then stopped by immersing the slides in distilled water. The sections were counterstained with toluidine blue, followed by dehydration in a series of graded ethanol, cleared in histolene and mounted in Ultramount 4 (Fronine) mounting medium. As negative controls, the primary antibody was omitted and these sections always appeared blank.

PVDF membranes were captured using a Canon IXUS Digital Camera. Tissue sections were viewed under an Olympus BX50 light microscope and photographed using and attached Olympus DP70 digital camera connected to Olympus DP controller software. Captured images were transferred into Adobe Photoshop 5.5. In some images the contrast and brightness were adjusted to obtain a light background. No other image manipulations were performed.

The Na, K-ATPase $\alpha_{1}$-subunit (the catalytic subunit [5]) was detected at all ages investigated from E15 onwards using both immunohistochemistry and Western Blot analysis (Figs. 1 and 2). Immunohistochemistry showed weak apical immunoreactivity at E15 in some of the 
epithelial cells in the lateral ventricular choroid plexus (Fig. 1A). The reactivity increased with age and the majority of the epithelial cells was positive around P9 (Fig. 1B). Western Blot analysis showed an increase the amount of NKA $\alpha_{1}$ with age, starting with barely visible levels at E15 (Fig. 2A).

The choroid plexus epithelial cells go through four stages in their development (Stage I-IV [24]). Briefly, Stage I cells are tall and appear pseudostratified and Stage II cells are low, columnar and contain abundant glycogen deposits, Stage III cells have less glycogen and Stage IV cells are cuboidal with no glycogen deposits. In the rat Stage I and II cells are present during embryonic development whereas Stage III and IV appear postnatally. Previous work has shown that AQP1 appears in a transitional stage between Stage I and Stage II when the epithelial cells are no longer pseudostratified, but do not yet contain glycogen [13]. NKA $\alpha_{1}$ appeared when the epithelial cells were in Stage II (Fig. 1A). However, epithelial cells in Stage II without $\mathrm{NKA} \alpha_{1}$ immunoreactivity were also present in the lateral ventricular choroid plexus at E15 indicating that no direct correlation between the stage of development and NKA $\alpha_{1}$ presence was evident. This shows that that $\mathrm{NKA} \alpha_{1}$ appears in the developing choroid plexus later than AQP1.

NKA $\alpha_{1}$ has not been previously investigated during early choroid plexus development, but the $\beta_{1^{-}}$and $\beta_{2^{-}}$-subunits (which are part of the ATPase complex in the choroid plexus [10]) have been found by immunohistochemistry to be present in the rat lateral ventricular choroid plexus from E15 [10]. The function of the $\beta$-subunits is not entirely clear, but they are thought to be involved in insertion in the membrane. The presence of the $\beta$ - and $\alpha$-subunits at E15, and especially the localisation of the $\alpha$-subunit in the membrane fraction indicate that the NKA complex is correctly assembled and functional in early development. However, due to the low levels of protein in embryonic compared to adult choroid plexus, it is unlikely that NKA activity can account for the movement of water required for the expansion of the ventricles occurring at this time. Recently, the importance of NKA in initial ventricular expansion was shown in Zebra fish with mutated NKA in which the ventricles formed but failed to expand [18]. It is however not clear if this is the case in mammals. Indeed in one study in the rat, NKA activity was shown to be 2-3 times lower during the first postnatal week than in the adult animals [23], suggesting that the activity at E15 might be substantially lower. However, a similar study in rabbits showed no change in NKA activity during development [21].

Using immunohistochemistry, CAII was not detected at E15 (Fig. 1C), E18 or P0. Cytosolic immunoreactivity was apparent at P9 (Fig. 1D), when most cells appeared immunopositive. Western Blot analysis showed the presence of CAII from E15 onwards, and an increase with age was observed (Fig. 2B). However, when the same amount of total protein was analysed from blood samples collected at P0, a clear band was visible, accounting for a proportion of the reactivity seen in the $\mathrm{P} 0$ choroid plexus sample (Fig. 2B). Although these samples are not directly comparable this shows that some of the immunoreactivity seen in the Western Blot analysis can be accounted for by blood contamination. Thus it is not possible to determine if CAII is or is not present in the epithelial cells at E15. However, if CAII is present, as suggested by the Western Blot analysis, it would be at very low levels, below the detection limits of the immunohistochemical procedure. No difference in immunoreactivity or localisation was seen after standard antigen retrieval procedures (data not shown). CAII immunoreactivity appeared sometime during Stage III of choroid plexus development.

Previous work has described CAII in the rat at E15 and in human from 7 weeks $[5,17,19]$. In one of the human studies, a decrease in the immunoreactivity was seen with age [17]. Further, in experiments measuring CSF secretion the use of the carbonic anhydrase inhibitor acetazolamide had no apparent effect [2]. The lack of inhibition could be due to the fact that the effect of acetazolamide was assessed by measuring the protein concentration in CSF. The 
assumption was that if CSF production is reduced, the bulk flow and re-absorption of CSF will also be reduced, leading to accumulation of protein. In accordance with this, rats from postnatal day 8 to adulthood showed an increase in the protein concentration in the CSF in response to acetazolamide, whereas no change was seen in newborn rats [10]. Based on these and later studies measuring the transfer of $\mathrm{I}^{125}$-albumin from blood into brain and CSF in newborn and juvenile rats it was concluded that CSF production was very low in the early postnatal period [1].

In the rat, gradients for $\mathrm{Na}^{+}, \mathrm{K}^{+}$and $\mathrm{Cl}^{-}$are present at or shortly after birth [1,9], but nothing appears to be known about embryonic stages in this species. The lack of effect of acetazolamide on newborn CSF protein concentrations may indicate that carbonic anhydrase driven CSF secretion is at a low level in neonatal rats (and presumably therefore also in the embryo). Thus other mechanisms are responsible for the formation of CSF that expands the cerebral ventricular system in embryonic development. In addition to the protein concentration-driven transfer of water [14], there may be other ion transfer mechanism present in embryonic choroid plexus such as the purinoceptors recently identified in the developing choroid plexus [11].

The present study has shown very low levels of both CAII and NKA in the rat choroid plexus at prenatal ages E15 and E18. During this period the cerebral ventricles more than double in size [12] and AQP1 water channels are already present in an adult-like pattern [13] implying secretion of fluid. The low levels of NKA and CAII during this period suggest that alternative mechanisms are likely to be involved. One possibility relates to the selective transfer of plasma proteins from blood into CSF in a subset of the epithelial cells, which appear to regulate the CSF protein concentration in development [14]. This protein transfer could be creating a colloid osmotic gradient which can drive the entry of water into CSF at a time when key adult enzymes involved in CSF secretion are only present in low levels. Evidence is lacking on whether or not functional ion gradients have been established as early as E15, when significant fluid transfer by the choroid plexuses occurs, however there may be specific mechanisms that contribute to CSF secretion early in brain development.

\section{Acknowledgements}

This work was supported by NIH grant R01NS043949-01A1.

\section{References}

1. Amtorp O. Transfer of $\mathrm{I}^{125}$-albumin from blood into brain and cerebrospinal fluid in newborn and juvenile rats. Acta Physiol Scand 1976;96:399-406. [PubMed: 1274618]

2. Amtorp O, Sørensen SC. The ontogenetic development of concentration differences for protein and ions between plasma and cerebrospinal fluid in rabbits and rats. J Physiol 1974;243:387-400. [PubMed: 4141371]

3. Bass NH, Lundborg P. Postnatal development of bulk flow in the cerebrospinal fluid system of the albino rat: clearance of carboxyl- $\left({ }^{14} \mathrm{C}\right)$ inulin after intrathecal infusion. Brain Res 1973;52:323-332. [PubMed: 4739806]

4. Brown PD, Davies SL, Speake T, Millar ID. Molecular mechanisms of cerebrospinal fluid production. Neuroscience 2004;129:957-970. [PubMed: 15561411]

5. Catala M. Embryonic and fetal development of structures associated with the cerebrospinal fluid in man and other species. Part I: the ventricular system, meninges and choroid plexuses. Arch Anat Cytol Pathol 1998;46:153-169. [PubMed: 9754371]

6. Davson, H.; Segal, MB. Physiology of the CSF and Blood-Brain Barriers. CRC Press; Boca Raton: 1996.

7. Desmond ME, Jacobson AG. Embryonic brain enlargement requires cerebrospinal fluid pressure. Dev Biol 1977;57:188-198. [PubMed: 863106] 
8. Dziegielewska KM, Ek J, Habgood MD, Saunders NR. Development of the choroid plexus. Microsc Res Tech 2001;52:5-20. [PubMed: 11135444]

9. Ferguson RK, Woodbury DM. Penetration of ${ }^{14} \mathrm{C}$-inulin and ${ }^{14} \mathrm{C}$-sucrose into brain, cerebrospinal fluid, and skeletal muscle of developing rats. Exp, Brain Res 1969;7:181-194. [PubMed: 5795246]

10. Gonzalez-Martinez LM, Avila J, Marti E, Lecuona E, Martin-Vasallo P. Expression of the betasubunit isoforms of the Na, K-ATPase in rat embryo tissues, inner ear and choroid plexus. Biol Cell 1994;81:215-222. [PubMed: 7696974]

11. Johansson PA, Burnstock G, Dziegielewska KM, Guida E, McIntyre P, Saunders NR. Expression and localization of $\mathrm{P} 2$ nucleotide receptor subtypes during development of the lateral ventricular choroid plexus of the rat. Eur J Neurosci 2007;25:3319-3331. [PubMed: 17553000]

12. Johansson PA, Dziegielewska KM, Ek CJ, Habgood MD, Liddelow SA, Potter AM, Stolp HB, Saunders NR. Blood-CSF barrier function in the rat embryo. Eur J Neurosci 2006;24:65-76. [PubMed: 16800861]

13. Johansson PA, Dziegielewska KM, Ek CJ, Habgood MD, Møllgård K, Potter A, Schuliga M, Saunders NR. Aquaporin-1 in the choroid plexuses of developing mammalian brain. Cell Tissue Res 2005;322:353-364. [PubMed: 16133142]

14. Johansson PA, Liddelow SA, Dziegielewska KM, Saunders NR. The blood-CSF barrier explainedwhen development is not immaturity. BioEssays 2008;30:237-248. [PubMed: 18293362]

15. Jones HC. Intercellular pores between the ependymal cells lining the roof of the fourth cerebral ventricle in mammalian fetuses. Surg Infancy Child 1980;31

16. Jones HC, Keep RF. The control of potassium concentration in the cerebrospinal fluid and brain interstitial fluid of developing rats. J Physiol 1987;383:441-453. [PubMed: 3656129]

17. Kida E, Palminiello S, Golabek AA, Walus M, Wierzba-Bobrowicz T, Rabe A, Albertini G, Wisniewski KE. Carbonic anhydrase II in the developing and adult human brain. J Neuropathol Exp Neurol 2006;65:664-674. [PubMed: 16825953]

18. Lowery LA, Sive H. Initial formation of zebrafish brain ventricles occurs independently of circulation and requires the nagie oko and snakehead/atp1a1a.1 gene products. Development 2005;132:20572067. [PubMed: 15788456]

19. Mani-Ponset L, Masseguin C, Davet J, Herbute S, Maurel D, Ghandour MS, Reiss-Bubenheim D, Guell A, Gabrion J. Effects of an 11-day spaceflight on the choroid plexus of developing rats. Brain Res Dev Brain Res 1997;99:187-200.

20. Masuzawa T, Ohta T, Kawamura M, Nakahara N, Sato F. Immunohistochemical localization of Na +, K+-ATPase in the choroid plexus. Brain Res 1984;302:357-362. [PubMed: 6329462]

21. Mitchell W, Kim CS, O’Tuama LA, Pritchard JB, Pick JR. Choroid plexus, brain and kidney Na+,K +-ATPase: comparative activities in fetal, newborn and young adult rabbits. Neurosci Lett 1982;31:37-40. [PubMed: 6289198]

22. Nielsen S, Smith BL, Christensen EI, Agre P. Distribution of the aquaporin CHIP in secretory and resorptive epithelia and capillary endothelia. Proc Natl Acad Sci USA 1993;90:7275-7279. [PubMed: 8346245]

23. Parmelee JT, Johanson CE. Development of potassium transport capability by choroid plexus of infant rats. Am J Physiol 1989;256:R786-R791. [PubMed: 2538088]

24. Shuangshoti S, Netsky MG. Histogenesis of choroid plexus in man. Am J Anat 1966;118:283-316. [PubMed: 5915034]

25. Siegel GJ, Holm C, Schreiber JH, Desmond T, Ernst SA. Purification of mouse brain (Na++ K+)ATPase catalytic unit, characterization of antiserum, and immunocytochemical localization in cerebellum, choroid plexus, and kidney. J Histochem Cytochem 1984;32:1309-1318. [PubMed: 6094658] 

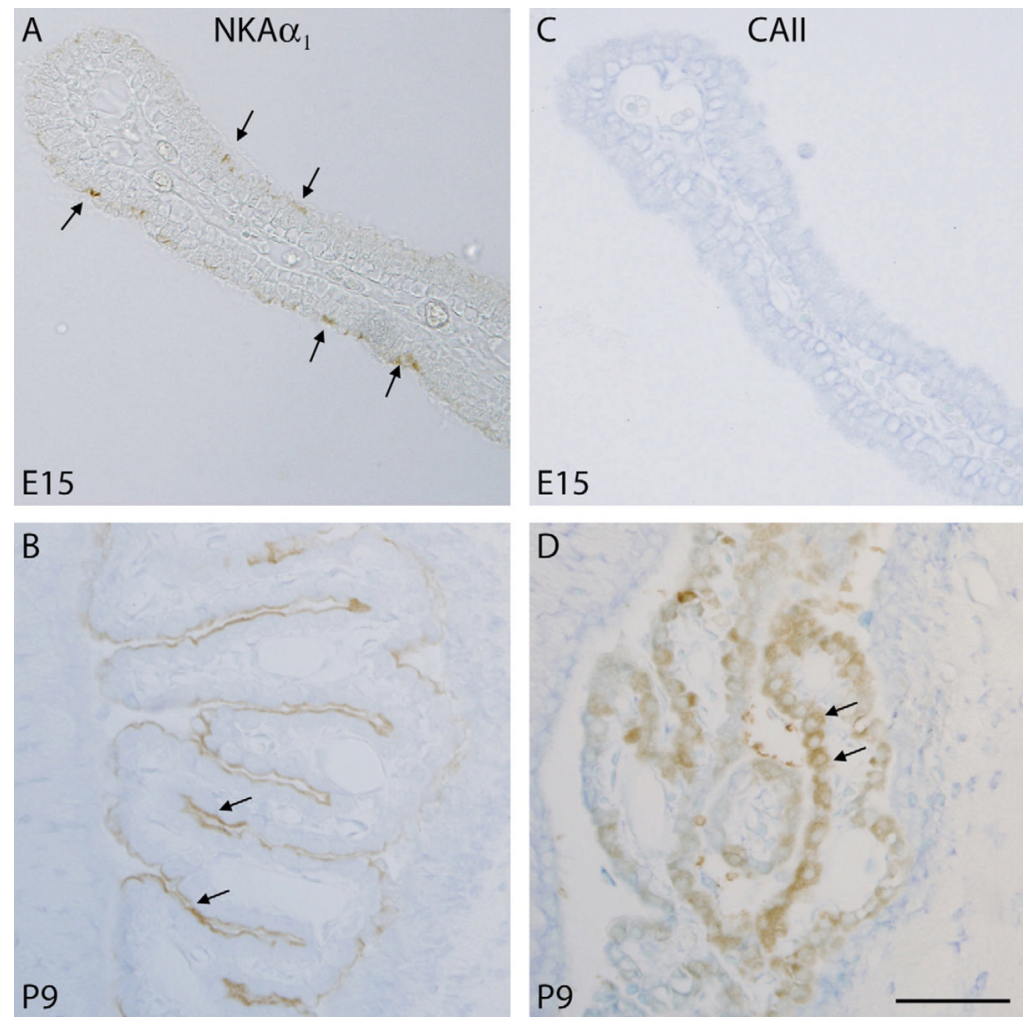

Fig. 1.

NKA $\alpha_{1}$ immunoreactivity (A-B) was present on in the choroid plexus at all ages examined from E15 to adult. AtE15 (A) only a few cells were positive (arrows) and the number of positive cells increased with age and at $\mathrm{P} 9$ (B) most cells were strongly immunopositive (arrows). The immunoreactivity was located exclusively on the apical membrane at all ages examined (E15, E18, P0, P9 and adult). CAII immunoreactivity (C-D) was not detected between E15 (C) and P0 (not illustrated), but was detected at P9 (D). At P9 the immunoreactivity was cytosolic and present in most of the epithelial cells (arrows). Sections were counterstained with toluidine blue. $E$ is the embryonic day, $P$ is the postnatal day, NKA $\alpha_{1}$ is the Na, K-ATPase $\alpha_{1}$-subunit and CAII is carbonic anhydrase II. Scale bar $50 \mu \mathrm{m}$. 

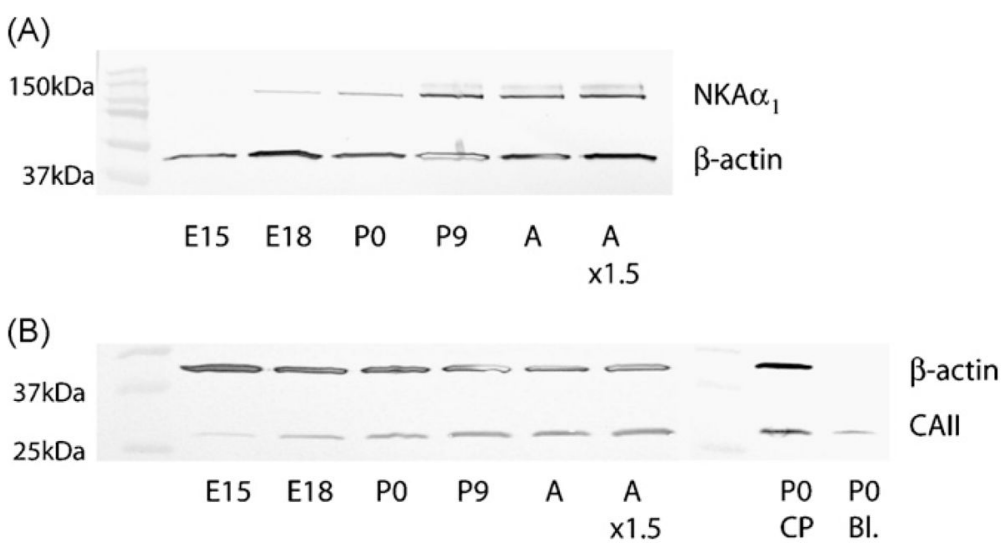

Fig. 2.

Western Blot analysis of NKA $\alpha_{1}$ and CAII during choroid plexus development in the rat. (A) The developmental profile of $\mathrm{NKA} \alpha_{1}$ in isolated lateral ventricular choroid plexus showed an increase in band intensity with increasing age. (B) The developmental profile of CAII showed a progressive increase in band intensity with age. CAII immunoreactivity was detected at all ages from E15. The signal from blood and choroid plexus samples containing the same amount of protein were compared. A distinct band was detected in the blood sample indicating that some of the signal from the highly vascularised choroid plexus might be due to blood contamination. $\boldsymbol{E}$ is the embryonic day, $\boldsymbol{P}$ is the postnatal day, A is adult, $\mathrm{NKA} \alpha_{1}$ is the $\mathrm{Na}$, $\mathrm{K}$-ATPase $\alpha$-subunit, CAII is carbonic anhydrase II, $\mathrm{A} \times 1.5$ represents loading of $50 \%$ more protein in this lane to show that the signal was not saturated, CP is choroid plexus and B1. is blood. 